\title{
Características epidemiológicas y clínicas de la leishmaniasis visceral en Paraguay de enero de 2008 a diciembre de 2014.
}

Rios-González Carlos Miguel ${ }^{1 a}$, Molinas Gómez Sandra Karina ${ }^{2 a}$, Gómez López Fredy Osmar ${ }^{2 a}$, Florentin Quintana Daisy Monserrat ${ }^{2 a}$, Sigmund Britez Claudia Jazmin ${ }^{2 a}$, Azuaga Franco Herminia María de las Nieves ${ }^{2 a}$, Canese-Krivoshein Pedro Andres ${ }^{2 b}$

\author{
Facultad de Ciencias Médicas, Universidad Nacional de Caaguazu, Cnel. Oviedo, Paraguay. \\ Facultad de Ciencias de la Salud, Universidad Privada del Este, Asunción, Paraguay. \\ Estudiante de Medicina Humana \\ Bioquímico. \\ Correo electrónico: carlosmigue_rios@live.com
}

Fecha de recibido: Noviembre 25 de 2015

Fecha de corrección: Febrero 23 de 2016

Fecha de aceptación: Marzo 13 de 2016

\section{Resumen:}

Objetivo: Determinar las características epidemiológicas y clínicas de la leishmaniasis visceral en Paraguay de enero de 2008 a diciembre de 2014 Material y Métodos: Estudio observacional, descriptivo tipo serie de casos de leishmaniasis visceral reportados por las regiones sanitarias al SENEPA durante los meses de enero del 2008 a diciembre del 2014. Los datos proveídos por fichas epidemiológicas fueron cargados en una planilla de Microsoft Office Excel 2013, diseñado para el efecto. Los datos se presentan en tablas de frecuencias, y medidas de tendencia central y dispersión. Resultados: fueron reportados 646 pacientes con leishmaniasis visceral, siendo la mediana de edad 23 años de edad y estuvo comprendida entre 2 años, 6 meses y 47 años, la edad mínima registrada fue de 1 mes y la máxima 87 años. 66,61\%(424), y $67,39 \%$ (436) fue del sexo masculino provino del departamento Central. Se encontró una coinfección con VIH en 6,66\% (40) de los casos. Conclusión: la leishmaniasis sigue siendo endémica en el país, siendo más frecuente de diagnóstico en el sexo masculino.

Palabra claves: Leishmaniasis; Leishmaniasis Visceral; Epidemiologia. Fuente: DeCS BIREME
Epidemiological and Clinical characteristics of visceral leishmaniasis in Paraguay January 2008 to December 2014 features.

\begin{abstract}
:
Objective: To determine the epidemiological and clinical visceral leishmaniasis in Paraguay January 2008 to December 2014 features. Material and Methods: Observational, descriptive series of cases of visceral leishmaniasis reported by the health regions to SENEPA during the months of January 2008 to December 2014. The data type provided by epidemiological records were loaded into a spreadsheet Microsoft Office Excel 2013 designed for the purpose. The data measures of central tendency and dispersion are presented in frequency tables and. Results: 646 patients were reported with visceral leishmaniasis, the median age being 23 years of age and was between 2 years, 6 months and 47 years, the minimum age recorded was 1 month and the maximum $87.66 .61 \%$ (424) and $67.39 \%$ (436) were male came from the Central department. HIV coinfection with $6.66 \%$ (40) cases was found. Conclusion: Leishmaniasis is still endemic in the country, being more frequent diagnosis in males.

Key word: Leishmaniasis; Visceral leishmaniasis; Epidemiology. Source: MeSH NLM
\end{abstract}

\section{Introducción:}

La leishmaniasis visceral americana, también llamado Kala Azar, es una enfermedad protozoaria causada por el L. infantum chagasi,(1), esta presenta una amplia distribución tropical (2), esta representa un problema de salud pública en muchos países del Tercer Mundo. La presentación clínica varía desde una infección asintomática o subclínica de la enfermedad sintomática grave y complicada.

Según la Organización Mundial de la Salud (OMS) se estima que cada año se producen 1,3 millones de nuevos casos y entre 20000 y 30000 defunciones, se encuentra vinculada a los cambios ambientales, como la deforestación, la construcción de presas, los sistemas de riego y la urbanización (3), por lo afecta a las poblaciones más pobres del planeta, está asociada a la malnutrición, los desplazamientos de población, las malas condiciones de vivienda, la debilidad del sistema inmunitario y la falta de recursos.

En el 2012 OMS dirigió una importante revisión de la incidencia de leishmaniasis en todo el mundo y concluyo que las tasas de letalidad pueden ser de $10 \%-20 \%$ incluso con el acceso al tratamiento (4).

"Es altamente endémica en el subcontinente indio y África oriental. Se estima que cada año se producen en el mundo entre 200000 y 400000 nuevos casos de leishmaniasis visceral, más del 90\% de ellos en seis países: Bangladesh, Brasil, Etiopía, India, Sudán y Sudán del Sur” (5). En América del Sur el primer caso de Leishmaniasis visceral fue descrito en el Paraguay en el año 1913 por el Dr. Migone (6)

En el 2013 en Paraguay se realizó un estudio sobre la seroprevalencia de anticuerpos IgG contra Leishmania chagasi en caninos, en este estudio se encontró una seroprevalencia poblacional del $26 \%$ (7).

Un reto importante en el manejo clínico de VL es la debilidad de los sistemas de salud en regiones endémicas de la enfermedad, en la actualidad no hay pruebas están actualmente disponibles que pueden detectar la infección asintomática o predecir la progresión de la infección a la enfermedad, 
asimismo es importante considerar el diagnóstico de coinfección con VIH es muy frecuente lo cual será un problema de salud pública en el futuro (8).

Los objetivos de estudio fueron: a) determinar las características epidemiológicas y clínicas de la leishmaniasis visceral en Paraguay de enero de 2008 a diciembre de 2014, b) describir la prevalencia de la infección por sexo y procedencia y c) identificar la coinfecciones asociadas a los pacientes con la leishmaniasis.

\section{Material y métodos}

\section{1- Diseño y Población del estudio:}

Se realizó un estudio observacional, descriptivo tipo serie de casos de leishmaniasis visceral reportados por las regiones sanitarias al Servicio Nacional de Erradicación del Paludismo (SENEPA) dependiente del Ministerio de Salud Pública y Bienestar Social durante los meses de enero del 2008 a diciembre del 2014.

La población la constituyeron 646 fichas epidemiológicas de pacientes reportados con leishmaniosis de 1 mes a 87 años de edad de ambos sexos durante los meses de meses de enero del 2008 a diciembre del 2014 (Figura 1).

- Criterios de inclusión: fichas epidemiológicas de pacientes reportados al SENEPA desde enero de 2008 a diciembre del 2014.

- Criterios de exclusión: Fueron excluidos dentro del estudio aquellas fichas que estaban completadas de manera inadecuada, aquellas fichas con notificación de sospechosos y aquellas fichas donde faltaban las variables de interés.

Se consideraron como Leishmaniosis positiva a los casos confirmados por las siguientes pruebas diagnósticas, observación directa del parásito, cultivo, PCR o serología con el antígeno RK39.

\section{2- Instrumento y método de recolección de datos.}

Para la recolección de los datos se diseñó un cuestionario tipo mixto divido en secciones, la primera donde se registraron las variables sociodemográficas (Edad, Sexo), la segunda variables epidemiológicas (Mes de notificación, Departamento de procedencia, Año de notificación), y la tercera las variables clínico-laboratoriales (Diagnostico de Leishmaniasis, Coinfecciones).

El principal posible sesgo es el sesgo de selección, siendo controlado este por un control cruzado por todos los investigadores del estudio.

\section{3- Procesamiento y análisis de datos}

Los datos proveídos por las fichas fueron cargadas en una planilla electrónica de Microsoft Office Excel 2013, y luego del control de calidad realizado mediante el control de filtros y la verificación con la fichas epidemiológicas, fueron exportados para su análisis en el programa estadístico R commander 3.0.1. Los posibles confusores pueden ser el sexo y la procedencia, para evitarlo se realizó un análisis estratificado.

Los datos se expresan en estadísticas descriptivas (medida de tendencia central y dispersión), tablas de frecuencias y proporciones.

\section{4- Aspectos éticos}

En esta investigación se pretende caracterizar la leishmaniasis visceral, objetivo que en concordancia con lo señalado en la declaración de Helsinki corresponde a una investigación que busca el beneficio de la población. Asimismo lo constituirá un análisis de fuentes secundarias, por lo que para la ejecución del estudio se contó con la aprobación del director general del SENEPA.

Se puede indicar que los principios de: autonomía, beneficencia, no maleficencia y justicia, consignados en la normatividad vigente son preservados en el desarrollo de la investigación. Para manejar los datos personales de los participantes, se tendrán en cuenta las siguientes consideraciones: se tomarán sólo los datos estrictamente necesarios para resolver los objetivos de investigación; no se tomarán datos personales que permitan la identificación de los participantes, esto con el fin de salvaguardar el derecho al anonimato.

\section{Resultados}

Grafico 1: Distribución de los notificados por Leishmaniosis por edad. Enero de 2008 a diciembre de 2014

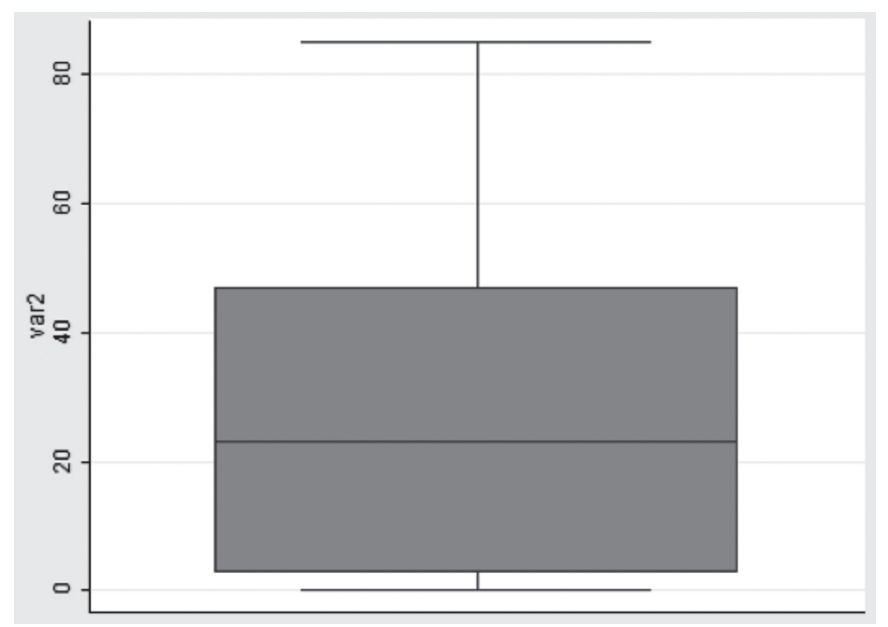

Gráfico 2. Distribución de los notificados por Leishmaniosis por edad enero de 2008 a diciembre de 2014

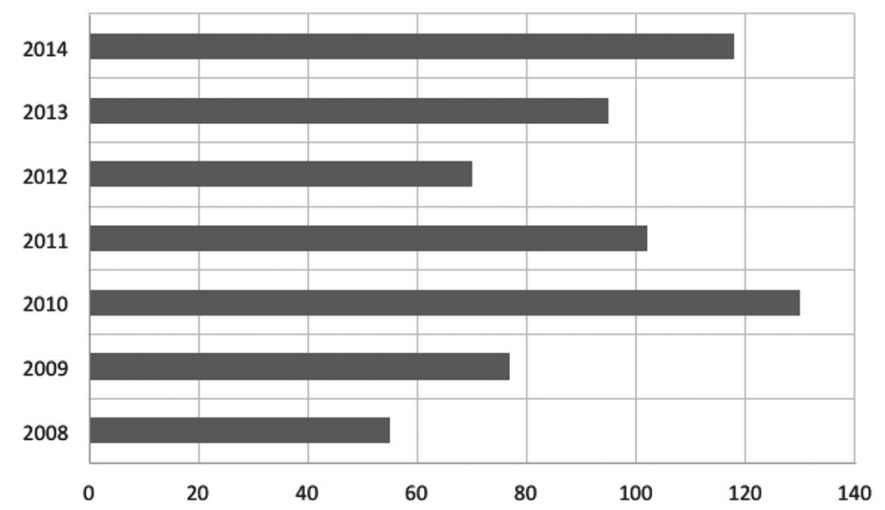

Durante el periodo de estudio fueron reportados 646 pacientes con leishmaniasis visceral, siendo la mediana de edad 23 años de edad y estuvo comprendida entre 2 años, 6 meses y 47 años, la edad mínima registrada fue de 1 mes y la máxima 87 años (Grafico 1).

Del total de la población de estudio, el 67,39\% (436) fue del sexo masculino. De acuerdo a la distribución de las por departamentos se encontró un 66,61\%(424) en departamento Central 10,40\%(67) en Cordillera y 6,37\%(41) en Asunción (Distrito Capital). 
De la totalidad de los diagnosticados por año, se ha detectado $20,09 \%(130)$ en el año 2010, 18,24\%(118) en el año 2014 y en el año 2011 un $15,77 \%$ (152), siendo diagnosticado en enero 13,93\% (90), 9,75\%(63) en abril, 9,29\% (60) en setiembre y 8,67\%(56) en el mes de mayo (Cuadro 1)

Cuadro 1: Características generales de los casos de leishmaniasis notificados. Enero de 2008 a diciembre de 2014.

\begin{tabular}{|c|c|c|}
\hline & $\%$ & $\mathrm{n}$ \\
\hline \multicolumn{3}{|l|}{ Sexo } \\
\hline Masculino & 67,39 & 436 \\
\hline Femenino & 32,61 & 211 \\
\hline \multicolumn{3}{|c|}{ Departamentos de procedencia } \\
\hline Concepción & 0,31 & 2 \\
\hline San Pedro & 1,55 & 10 \\
\hline Cordillera & 10,40 & 67 \\
\hline Guaira & 1,55 & 10 \\
\hline Caaguazú & 0,78 & 5 \\
\hline Caazapá & 0,31 & 2 \\
\hline Itapuá & 3,88 & 25 \\
\hline Misiones & 0,62 & 4 \\
\hline Paraguarí & 6,06 & 39 \\
\hline Alto Paraná & 0,47 & 3 \\
\hline Central & 66,61 & 424 \\
\hline Ñeembucú & 0 & 0 \\
\hline Amambay & 0,47 & 3 \\
\hline Canindeyú & 0,16 & 1 \\
\hline Presidente Hayes & 0,47 & 3 \\
\hline Boquerón & 0 & 0 \\
\hline Alto Paraguay & 0 & 0 \\
\hline Asunción (Distrito capital) & 6,37 & 41 \\
\hline \multicolumn{3}{|l|}{ Año de notificación } \\
\hline $1-2008$ & 8,50 & 55 \\
\hline $2-2009$ & 11,90 & 77 \\
\hline $3-2010$ & 20,09 & 130 \\
\hline $4-2011$ & 15,77 & 102 \\
\hline $5-2012$ & 10,82 & 70 \\
\hline $6-2013$ & 14,68 & 95 \\
\hline $7-2014$ & 18,24 & 118 \\
\hline \multicolumn{3}{|l|}{ Mes de notificación } \\
\hline Enero & 13,93 & 90 \\
\hline Febrero & 7,12 & 46 \\
\hline Marzo & 8,67 & 56 \\
\hline Abril & 9,75 & 63 \\
\hline Mayo & 8,67 & 56 \\
\hline Junio & 6,35 & 41 \\
\hline Julio & 6,66 & 43 \\
\hline Agosto & 6,35 & 41 \\
\hline Setiembre & 9,29 & 60 \\
\hline Octubre & 7,74 & 50 \\
\hline Noviembre & 7,12 & 46 \\
\hline Diciembre & 8,36 & 54 \\
\hline
\end{tabular}

En cuanto a las características de la infección, se ha encontrado $6,66 \%$ (40) coinfección con el VIH, y 38,88\% (243) infección asociado a enfermedades respiratorias.
9,13\% (55) de los pacientes diagnosticados han fallecido a causa de la leishmaniasis (Cuadro 2).

Cuadro 2: Distribución de los notificados por características clínicas. Enero de 2008 a diciembre de 2014

\begin{tabular}{lcc}
\hline & $\%$ & $\mathrm{n}$ \\
\hline Coinfeccion con VIH & & \\
$\mathrm{Si}$ & 6,66 & 40 \\
$\mathrm{No}$ & 93,34 & 560 \\
Coinfeccion con infecciones respiratorias & & \\
$\mathrm{Si}$ & 38,88 & 243 \\
$\mathrm{No}$ & 61,12 & 382 \\
Muerte por leishmania & & \\
$\mathrm{Si}$ & 9,13 & 55 \\
No & 90,86 & 547 \\
Muerte por otras causas & & \\
$\mathrm{Si}$ & 0,91 & 5 \\
No & 99,09 & 543 \\
\hline
\end{tabular}

\section{Discusion}

"La Leishmaniosis Visceral, en general, endémica en 98 países y territorios y es responsable de una carga de la enfermedad de 2,35 millones de AVAD (años de vida ajustados por discapacidad), de los cuales 2,3\% ocurren en las Américas" $(9,10,11)$ en el país es endémica, registrándose la mayor proporción de los casos en un área geográfica que abarca al distrito de Asunción y a los departamentos de Central, Paraguarí y Cordillera, probablemente debido a la instalación del ciclo de transmisión de la enfermedad, con un gran crecimiento urbano desordenado de la población, lo cual coincide a medida que pasa los años con los datos manejados por SENEPA del Ministerio de Salud Pública y Bienestar Social, que a pesar de ser considerada patología de denuncia obligatoria muchos son los casos que no son remitidos al servicio (12).

El año de mayor frecuencia de esta patología ha sido el año 2010 donde se ha registrado $20,09 \%$ del total de los casos registrados, así como el año 2014, donde se registraron 18,24\%, asimismo al comparar los datos del mismo sistema se ve un aumento a medida que pasan los años como poder ser apreciado en el grafico 2 .

Los resultados del presente estudio la edad mínima de presentación de la leishamnia fue de 1 mes y la máxima de 87 años, ambos en los extremos de la vida, donde la tasa de mortalidad de esta patología es altísima (13).

Según los datos manejados por la OMS, la Leishmania y VIH se produce en 35 países, por lo que aumenta la carga de la enfermedad debido a la mayor dificultad en la gestión y el tratamiento clínico $(14,15)$, siendo el estudio un $6,66 \%$ de esta confección, en países de África esta asociación es imperativa, sin en Latinoamerica la prevalencia de coinfeccion no es tan alta $(15,16)$, asimismo se ha visto casos sepsis bacteriana graves con derivaciones fatales (17) y casos asintomáticos en pacientes con trasplante hepático (18).

La limitación de estudio se centra en que provino de una fuente secundaria, siendo de esta manera eliminadas mucho de los datos por la falta del cumplimiento del criterio de inclusión. Son sumamente escasos los estudios sobre esta patología en el país, por lo que el conocimiento sobre estado actual de esta patología es relativa, es imperativo la ejecución de proyectos de investigación que exploren 
la seroprevalencia canina por lo que constituyen el principal factor de riesgo para el ser humano $(19,20)$, el mapeo correspondiente de las áreas endémicas y la implementación de estrategias de intervención en estas áreas.

Al finalizar la investigación se puede concluir que los departamentos endémicos siguen siendo Central y Cordillera, asimismo que los meses de mayor reporte son enero, abril y setiembre, cabe recalcar una gran frecuencia de asociación con HIV e infecciones respiratorias. Si bien son muchas las limitaciones y los resultados presentados en el estudio no son muchos, es importante destacar que estudios sobre esta patología en el país son escasos e incluso los datos manejados por las autoridades sanitarias son escasas.

\section{Conflicto de interés:}

Los autores declaran no tener conflictos de interés. Autofinanciado por los autores.

\section{Referencias}

1. Ready P. Epidemiology of visceral leishmaniasis. Clin Epidemiol. 2014; 6:147-54

2. Mansueto P, Seidita A, Vitale G, Cascio A. Leishmaniasis in travelers: a literature review. Travel Med Infect Dis. 2014;12(6 Pt A):563-81

3. Organización Mundial de la Salud [sede Web]. Enfermedades transmitidas por vectores. [acceso 22 de febrero de 2016]. Disponible en: http://www.who.int/mediacentre/factsheets/ fs387/es/

4. Organización Mundial de la Salud [sede Web]. Leishmaniasis. Nota de Prensa Febrero 2015. [acceso 22 de febrero de 2016]. Disponible en: http://www.who.int/mediacentre/factsheets/ fs $375 /$ es/

5. Pace D. Leishmaniasis. J Infect. 2014;69 Suppl 1:S10-8

6- Arbo A. Leishmaniasis Visceral en Paraguay. Revista de Medicina Tropical. 2014.

7. Portillo V, Benítez R., Acosta L. Prevalencia de leishmaniasis visceral canina en el área de influencia de la Unidad de Salud de la Familia Marín Ka’aguy, Luque. Revista de Salud Pública del Paraguay. 2013; 1(2); 11-18.

8. Singh O, Sundar S. Developments in Diagnosis of Visceral Leishmaniasis in the Elimination Era. J Parasitol Res. 2015.

9. Organización Mundial de la Salud [sede Web]. Control de Leishmaniasis: informe de una reunión del Comité de Expertos de la OMS sobre el Control de las Leishmaniasis, OMS, Italia; 2012. Disponible en: http://apps.who.int/iris/bitstream/10665/82766/1/ WHO_TRS_949_spa.pdf

10. Alvar J, Velez I, Bern C, Herrero M, Desjeux P, Cano J. Leishmaniasis worldwide and global estimates of its incidence. PloS one. 2012; 7(5): e35671.

11. Ready PD. Epidemiology of visceral leishmaniasis. Clin Epidemiol. 2014; 6(6): 147-154.

12. Instituto Nacional de Salud. Protocolo de Vigilancia Epidemiológica: Leishamniasis. MINSALUD. Colombia; 2014.
13. Ministerio de Salud Pública y Bienestar Social. Manual de Diagnóstico y Tratamiento: Leishmaniasis. MSPBS, Paraguay. 2011.

14. Vilas V, Maia-Elkhoury A, Yadon Z, Cosivi O, Sanchez-Vazquez M. Visceral leishmaniasis: a One Health approach. Veterinary Record. 2014; 175(2): 42-44.

15. Organización Mundial de la Salud. Leishmaniasis. Nota de Prensa Abril 2015. Disponible en: www.paho.org/leishmaniasis

16. Hailu A, Dagne D, Boelaert M . Leishmaniasis. In Neglected Tropical Diseases-Sub-Saharan Africa (pp. 87-112). Springer International Publishing. 2016.

17. Diro E, Lynen L, Ritmeijer K, Boelaert M, Hailu A, van Griensven J. Visceral Leishmaniasis and HIV coinfection in East Africa. Plos Neglected Tropical Diseases [serial on the Internet]. (2014, June 26), [cited February 11, 2016]; 8(6): e2869

18. Endris M, Takele Y, Woldeyohannes D, Tiruneh M, Mohammed R, Diro E, et al. Bacterial sepsis in patients with visceral leishmaniasis in Northwest Ethiopia. Biomed Research International [serial on the Internet]. (2014), [cited February 11, 2016]; 2014361058

19. Clemente W, Rabello A, Faria L, Peruhype-Magalhães V, Gomes L, Romanelli R, et al. High prevalence of asymptomatic Leishmania spp. infection among liver transplant recipients and donors from an endemic area of Brazil. American Journal Of Transplantation [serial on the Internet].2014, [cited February 17, 2016]; 14(1): 96101

20. Pimentel D, Ramos R, Santana M, Maia C, Carvalho G, Alves $\mathrm{L}$, et al. Prevalence of zoonotic visceral leishmaniasis in dogs in an endemic area of Brazil. Revista Da Sociedade Brasileira De Medicina Tropical [serial on the Internet]. 2015, [cited February 10, 2016]; 48(4): 491-493 